\title{
Method of lines for parabolic functional differential equations on cylindrical domains
}

\author{
H. Leszczyński · M. Matusik
}

Received: 29 March 2014 / Accepted: 27 December 2014 / Published online: 3 February 2015

C The Author(s) 2015. This article is published with open access at Springerlink.com

\begin{abstract}
The numerical method of lines is a technique for solving partial differential equations by discretising in all but one dimension. In this paper the solution of the approximate problem is extended outside the domain using the boundary condition. This leads to functional differential-algebraic equations. Sufficient conditions for the well-posedness, stability and convergence of the resulting method of lines are given.
\end{abstract}

Keywords Method of lines - Functional differential-algebraic equations - Robin boundary conditions

Mathematics Subject Classification $\mathrm{MSC} 34 \mathrm{~K} 10 \cdot \mathrm{MSC} 65 \mathrm{~N} 40$

\section{Introduction}

The method of lines (MOL) is a computational approach for solving PDE problems of the form

$$
\partial_{t} u=f\left(t, x, u, \partial_{x} u, \partial_{x x}^{2} u\right) .
$$

The numerical solution process proceeds in two steps:

1. Space semi-discretization (using, for example, finite differences).

H. Leszczyński $(\bowtie) \cdot$ M. Matusik

Institute of Mathematics, University of Gdańsk, Wit Stwosz 57, 80-952 Gdańsk, Poland

e-mail: mathl@univ.gda.pl; hleszcz@mat.ug.edu.pl

M. Matusik

e-mail:mnetka@mat.ug.edu.pl 
2. The resulting system of semi-discrete ordinary differential equations (ODE's) is integrated in time.

From the numerous literature concerning the numerical method of lines for classical differential equations we mention the monographs [8,25,27]. MOL for nonlinear parabolic functional differential equations with initial boundary conditions of the Dirichlet type are investigated in [30], where the error estimates implying the convergence of MOL on a rectangular domain is given. Shakeri and Dehghan [24] present two forms of MOL for one-dimensional nonlocal hyperbolic partial differential equation. MOL is used in [4] to obtain numerical solutions to a quasilinear parabolic inverse problem. Parabolic inverse problems can be reduced to a system of ODE's by fourth order compact scheme (see [18]).

The aim of the paper is to construct a method of lines for nonlinear parabolic functional differential equations with general initial boundary conditions and with a non-rectangular domain. To deal with a cylindrical domain, we can proceed in several ways. For instance, we can consider the polar coordinate system, like in $[25,27]$ or the Cartesian coordinate system. In the later case we have at least two possibilities. We can add extra points either at the boundary of the domain (see [14,17]), or outside the domain (see [19]). In this paper we consider the second possibility. In our scheme we obtain additional points using the reflection with respect to the boundary. We extend the solution of the approximate problem outside the domain by a transformation generated by the boundary condition. This leads to differential-algebraic equations (DAE). The theoretical analysis of DAE's and some appropriate numerical methods for initial and boundary value problems can be found in $[3,15]$. In [6] the authors study numerical solutions of DAE's. Soltaniana et al. [26] present a homotopy perturbation method to solve DAE's. The numerical solution of DAE's by the Rosenbroch onestage scheme with complex coefficients are investigated in [2]. However, there are considered systems which depend only on present time. Equations with aftereffects of various kinds (such as delays) are called functional differential equations (FDE) The theory of FDE's can be found in [7]. Semiexplicit numerical methods of the Rosenbroch type for functional differential-algebraic equations in the whole space were studied in [10]. In [13] the authors, on the basis of Newton's method, propose a fast quasilinearization numerical scheme, coupled with Rothe's method, for fully nonlinear parabolic equations. Comparison theorems for ODE and DAE systems are investigated in [11].

We admit some advantages of such scheme:

1. Simplicity of description and implementation.

2. No need to multiply cases.

3. No need to look for the nearest points.

4. No need to approximate boundary conditions.

5. A universal interpolation pattern and the values of $u$ in the grid points outside the domain $Q$.

The new approach is applicable for $n$-dimensional spatial variables, even though the numerical experiments are implemented for a two-dimensional spatial case. The weak point of the proposed method is a limitation on the boundary of the area of the spatial variable. Namely, we assume that the domain $\Omega$ is bounded, convex with the boundary 
of class $C^{2+\alpha}, 0<\alpha<1$. We require this assumption to construct a suitably regular extension of the functions beyond the domain $Q$. Fakhar-Izadi and Dehghan [5] deal with irregular domains for a weakly parabolic partial integro-differential equation. They propose a spectral method to find numerical solutions. It seems that spectral methods are more time consuming compared with methods on regular meshes which obey a kind of maximum principle.

Our technique may be used in many physical problems such as chemical reaction [9] and heat conduction [22], where a flow across the boundary surface is proportional to the difference between the surrounding density and the density inside surface. In these papers authors consider complex approximate methods, exclusively for the differential equations without any delay. In the article [23] one examines non-linearities and delays with Robin boundary conditions. In our paper we deal with difference schemes for functional differential equations with Robin boundary conditions, which is a much wider class that delays. We can also use our technique to a quasilinear parabolic system of PDE's, more precisely in the chemotaxis model considered in [29]. Recent advances in computational mathematical biology confirm our interest in theoretical investigations, compare: [16]. If the domain is not convex, as it often happens in realworld applications (chemotaxis, reaction-diffusion etc.), it is still possible to use our techniques by means of some smooth mappings which transform an irregular domain to a disc. For instance, a bean-shape region can be just projected to a disc.

The paper is organized in the following way. In Sect. 2 we set up the notation and terminology. Section 3 contains auxiliary lemmas. In Sect. 4 our main results are stated and proved. In the last section numerical experiments are presented.

\section{Formulation of the problem}

Let $\Omega \subset \mathbb{R}^{n}$ be a bounded, convex domain with boundary $\partial \Omega$ of class $C^{2+\alpha}$. Write

$$
Q_{0}=\left[-T_{0}, 0\right] \times \bar{\Omega}, \quad Q=[0, T] \times \bar{\Omega},
$$

where $T>0$ and $T_{0} \in \mathbb{R}_{+}=[0,+\infty)$. For a function $u: Q_{0} \cup Q \rightarrow \mathbb{R}$ and for $t \in[0, T]$ we define a function $u_{t}: Q_{0} \rightarrow \mathbb{R}$ by

$$
u_{t}(\tau, x)=u(t+\tau, x), \quad(\tau, x) \in Q_{0} .
$$

For any metric spaces $X$ and $Y$ we denote by $C(X, Y)$ the space of all continuous functions defined on $X$ and taking values in $Y$. In the case $Y=\mathbb{R}$ we write $C(X)$.

Given $f: Q \times C\left(Q_{0}\right) \times \mathbb{R}^{n} \rightarrow \mathbb{R}, a_{i j}: Q \rightarrow \mathbb{R}, \beta:[0, T] \times \partial \Omega \rightarrow[0,+\infty)$, $\psi: Q_{0} \rightarrow \mathbb{R}$, we consider the functional differential equation

$$
\partial_{t} u-\sum_{i, j=1}^{n} a_{i j}(t, x) \partial_{x_{i} x_{j}}^{2} u=f\left(t, x, u_{t}, \partial_{x} u\right), \quad(t, x) \in Q
$$

with the initial boundary conditions 


$$
\begin{gathered}
\frac{\partial u}{\partial n}+\beta(t, x) u=0, \quad(t, x) \in[0, T] \times \partial \Omega, \\
u(t, x)=\psi(t, x), \quad(t, x) \in Q_{0},
\end{gathered}
$$

where $n=n(x)$ is the unit outward normal on $\partial \Omega$.

Equation (1) with a particular right hand side can be interpreted as a reactiondiffusion equation, which is widely used as a model describing various physical, chemical and biological problems, see $[1,16,20]$. Note that the right hand side of the equation contains a functional variable. Therefore, we can also consider differential equation with deviated variables or differential integral equations, as it is shown in our examples 1 and 2 .

We transform the boundary condition (2) by considering an extension of a function $u$ outside $\tilde{Q}:=Q_{0} \cup Q$. Set $R(x)=x$ for $x \in \Omega$ and

$$
R(x)=\underset{\bar{x} \in \partial \Omega}{\operatorname{argmin}}\|\bar{x}-x\| \text { for } x \in \Omega^{c} .
$$

Given $u: \tilde{Q} \rightarrow \mathbb{R}$ and denoting $r(x)=2 R(x)-x$, we extend $u$ to the set $\left[-T_{0}, T\right] \times \mathbb{R}^{n}$ by

$$
u(t, x)=u(t, r(x)) \exp \{-\|x-r(x)\| \cdot \beta(t, R(x))\} \text { for } x \in \Omega^{c} .
$$

For any smooth function $u$ this extension implies boundary condition (2) on $[0, T] \times$ $\partial \Omega$. If $\beta \equiv 0$, then this extension is a mirror reflection with respect to the boundary $\partial \Omega$, generated by Neumann's conditions.

\section{Discretization}

We construct a regular mesh on $\mathbb{R}^{n}$ in the following way. Let $h=\left(h_{1}, \ldots, h_{n}\right), h_{i}>0$ be the steps of the mesh. For $m \in \mathbb{Z}^{n}, m=\left(m_{1}, \ldots, m_{n}\right)$, we denote nodal points in the following way: $x^{(m)}=\left(m_{1} h_{1}, \ldots, m_{n} h_{n}\right)$. Write $\mathbb{R}_{h}^{n}=\left\{x^{(m)}: m \in \mathbb{Z}^{n}\right\}$, $\Omega_{h}=\Omega \cap \mathbb{R}_{h}^{n}$ and

$$
\begin{aligned}
& \Omega_{h}^{*}=\left\{x^{(m)} \in \mathbb{R}_{h}^{n} \backslash \Omega_{h}: \underset{x^{(\bar{m})} \in \Omega_{h}}{\forall} \max _{i}\left|m_{i}-\bar{m}_{i}\right| \leq 1\right\}, \\
& Q_{h}=[0, T] \times \Omega_{h}, \quad Q_{h}^{*}=[0, T] \times \Omega_{h}^{*}, \quad Q_{0 . h}=\left[-T_{0}, 0\right] \times\left(\Omega_{h}^{*} \cup \Omega_{h}\right) .
\end{aligned}
$$

Denote $\tilde{\Omega}_{h}=\Omega_{h}^{*} \cup \Omega_{h}$. Let $p^{*}$ be the number of all nodal points of $\Omega_{h}^{*}$ and $p$ - the number of all nodal points of $\Omega_{h}$. Set $P=p^{*}+p$, see Fig. 1 . For any spaces $X$ and $Y$ we denote by ${ }^{X} Y$ the class of all functions defined on $X$ and taking values in $Y$. Difference operators for spatial variables are defined in the following way. Write $J=$ $\{(i, j): i, j=1, \ldots, n, i \neq j\}$. Suppose that we have two disjoint sets $J_{+}, J_{-} \subset J$ 
- differential equations

- algebraic equations

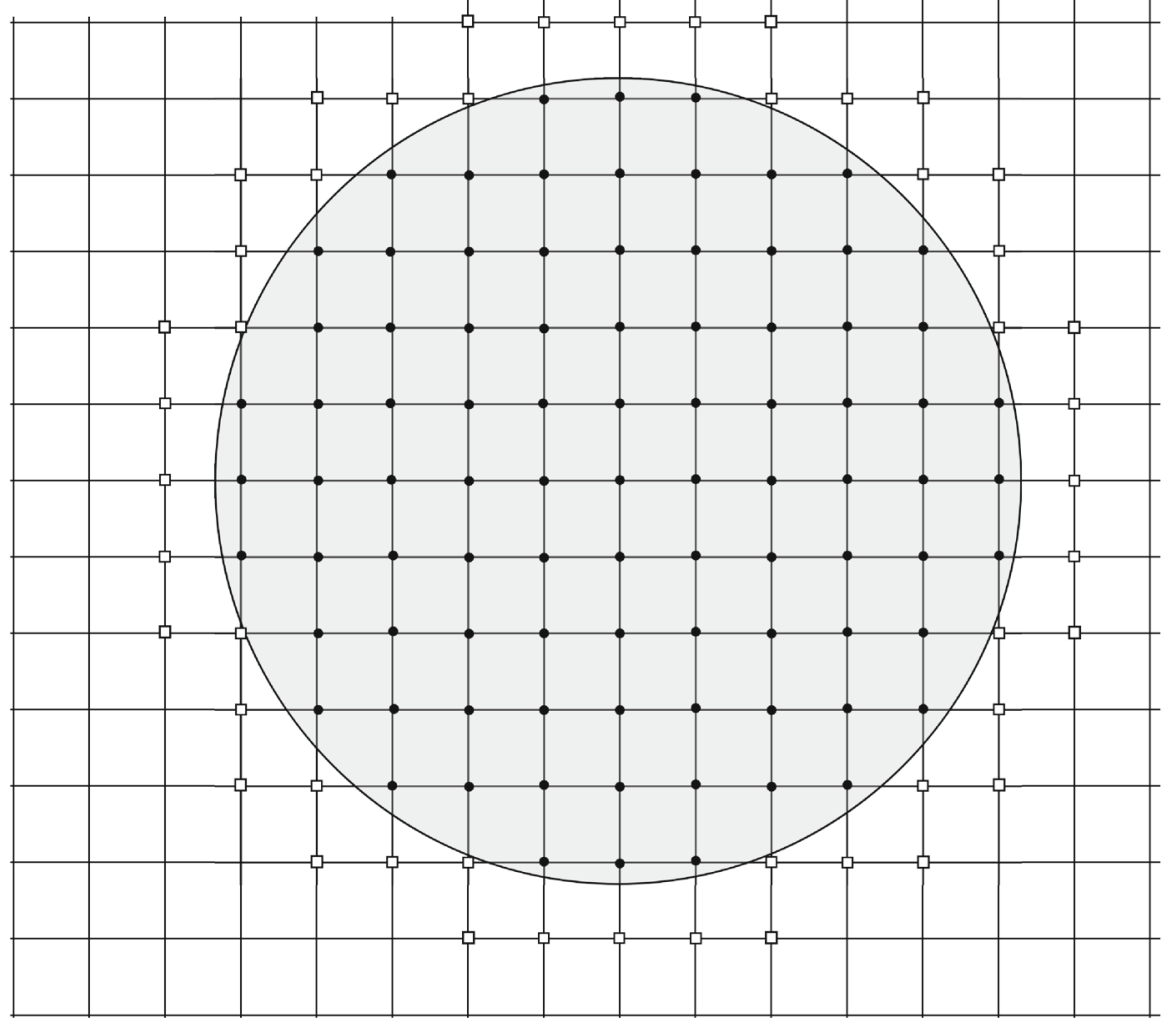

Fig. 1 Nodal points of $\Omega_{h}^{*}$ and $\Omega_{h}$

such that $J_{+} \cup J_{-}=J$ and $(i, j) \in J_{+}$if $(j, i) \in J_{+}$. Given $u:\left[-T_{0}, T\right] \rightarrow \tilde{\Omega}_{h} \mathbb{R}$ and $m \in \mathbb{Z}^{n}$. Write

$$
\begin{aligned}
\delta_{i}^{+} u^{(m)}(t) & =\frac{u^{\left(m+e_{i}\right)}(t)-u^{(m)}(t)}{h_{i}}, \delta_{i}^{-} u^{(m)}(t)=\frac{u^{(m)}(t)-u^{\left(m-e_{i}\right)}(t)}{h_{i}}, \\
\delta_{i} u^{(m)}(t) & =\frac{1}{2}\left[\delta_{i}^{+} u^{(m)}(t)+\delta_{i}^{-} u^{(m)}(t)\right], \\
\delta u^{(m)}(t) & =\left(\delta_{1} u^{(m)}(t), \ldots, \delta_{n} u^{(m)}(t)\right) .
\end{aligned}
$$

The difference operators $\delta^{(2)}=\left[\delta_{i j}\right]_{i, j=1, \ldots, n}$, are defined in the following way:

$$
\delta_{i i}^{(2)} u^{(m)}(t)=\delta_{i}^{+} \delta_{i}^{-} u^{(m)}(t) \text { for } i=1, \ldots, n
$$

and 


$$
\begin{aligned}
& \delta_{i j}^{(2)} u^{(m)}(t)=\frac{1}{2}\left[\delta_{i}^{+} \delta_{j}^{-} u^{(m)}(t)+\delta_{i}^{-} \delta_{j}^{+} u^{(m)}(t)\right] \quad \text { for }(\mathrm{i}, \mathrm{j}) \in \mathrm{J}^{-}, \\
& \delta_{i j}^{(2)} u^{(m)}(t)=\frac{1}{2}\left[\delta_{i}^{+} \delta_{j}^{+} u^{(m)}(t)+\delta_{i}^{-} \delta_{j}^{-} u^{(m)}(t)\right]
\end{aligned}
$$

Let us consider the interpolating operator

$\mathcal{J}_{h}: C\left(Q_{0 . h} \cup Q_{h} \cup Q_{h}^{*}\right) \rightarrow C\left(\operatorname{conv}\left(Q_{0 . h} \cup Q_{h} \cup Q_{h}^{*}\right)\right)$ defined by

$$
\mathcal{J}_{h}[u](t, x)=\sum_{s \in S_{+}} u^{(m+s)}(t)\left(\frac{x-x^{(m)}}{h}\right)^{s}\left(\mathbf{1}-\frac{x-x^{(m)}}{h}\right)^{1-s}
$$

where $x^{(m)} \leq x \leq x^{(m+\mathbf{1})}, \mathbf{1}:=(1, \ldots, 1)$,

$$
S_{+}=\left\{s=\left(s_{1}, \ldots, s_{n}\right): s_{i} \in\{0,1\}, 1 \leq i \leq n\right\} .
$$

In [12] (page 85) we find another extrapolation method. It is easy to see that $\mathcal{J}_{h}[u] \in$ $C\left(\operatorname{conv}\left(Q_{0 . h} \cup Q_{h} \cup Q_{h}^{*}\right)\right)$ and the norm of $\mathcal{J}_{h}$ is equal to 1 .

Consider the differential-difference equations

$$
\begin{aligned}
& \frac{d}{d t} u^{(m)}(t)-\sum_{i, j=1}^{n} a_{i j}\left(t, x^{(m)}\right) \delta_{i j}^{(2)} u^{(m)}(t) \\
& \quad=f\left(t, x^{(m)},\left(\mathcal{J}_{h}[u]\right)_{t}, \delta u^{(m)}(t)\right.
\end{aligned}
$$

for $\left(t, x^{(m)}\right) \in[0, T] \times \Omega_{h}$, the algebraic equations

$$
u^{(m)}(t)=\exp \left\{-\left\|x^{(m)}-r\left(x^{(m)}\right)\right\| \beta\left(t, R\left(x^{(m)}\right)\right\}\right) \mathcal{J}_{h}[u]\left(r\left(x^{(m)}\right)\right)
$$

for $\left(t, x^{(m)}\right) \in\left[-T_{0}, T\right] \times \Omega_{h}^{*}$, and with the initial condition

$$
u^{(m)}(t)=\psi\left(t, x^{(m)}\right)
$$

for $\left(t, x^{(m)}\right) \in\left[-T_{0}, 0\right] \times \Omega_{h}$.

The method of lines (5)-(7) can be written as the abstract differential-algebraic problem

$$
\left[\begin{array}{cc}
I_{p \times p} & 0 \\
0 & 0
\end{array}\right] \frac{d}{d t} \mathbf{u}-\mathbf{K u}=\varphi_{h}\left(t, \mathbf{u}_{t}\right)
$$

with the initial condition

$$
\mathbf{u}(t)=\psi_{h}(t), \quad \text { for } t \in\left[-T_{0}, 0\right] .
$$

$\mathbf{K}$ is generated by $\left[a_{i j}\right]$ and $\partial_{q_{j}} f$, while $\varphi_{h}$ by $f$. The choice is not unique. Here one can choose $K$ dependent on $a_{i j}$ for the components with the indices not greater than $p$ and $\varphi_{h}\left(t, \mathbf{u}_{t}\right)$ dependent on $f$. 


\section{Assumption A $[\sigma]$}

- $\sigma:[0, T] \times \mathbb{R}_{+} \rightarrow \mathbb{R}_{+}$is continuous non-decreasing in the second variable, $\sigma(t, 0)=0$, and the only maximal solution $\omega(\cdot ; \gamma, \tilde{\gamma})$ to the Cauchy problem

$$
\omega^{\prime}(t)=\sigma(t, \omega(t))+\tilde{\gamma}, \quad \omega(t)=\gamma \text { for } t \in\left[-T_{0}, 0\right]
$$

tends to zero as $\tilde{\gamma}, \gamma \rightarrow 0$.

In particular $\omega^{\prime}(t)=\sigma(t, \omega(t)), \omega(t)=0$ for $t \in\left[-T_{0}, 0\right]$ implies $\omega(\cdot ; 0,0) \equiv 0$.

\section{Assumption A[K]}

- K: $[0, T] \rightarrow M_{P \times P}$ is bounded and continuous,

$-k_{i 1}+\ldots+k_{i P}=0$ for each $i=1, \ldots, P$,

$-k_{i j} \geq 0$ for $i \neq j, i, j=1, \ldots, P$,

- the matrix $\mathbf{K}$ is DA-irreducible (i.e. $k_{i 1}+\ldots+k_{i p}>0$ for $i>p$ ).

Assumption A[C] The initial function $\psi_{h} \in C\left(\left[-T_{0}, 0\right], \mathbb{R}^{P}\right)$ satisfies the consistency condition

$$
K_{3} \psi_{h . D}(0)+K_{4} \psi_{h . A}(0)+\tilde{\varphi}_{A}(0)=0
$$

Lemma 1 Suppose that $\mathbf{A}[\mathbf{K}], \mathbf{A}[\sigma]$ is satisfied and $\tilde{\varphi}$ is bounded and continuous. Then problem

$$
\left[\begin{array}{cc}
I_{p \times p} & 0 \\
0 & 0
\end{array}\right] \frac{d}{d t} \boldsymbol{z}-\boldsymbol{K} z=\tilde{\varphi}(t)
$$

with the initial condition $z(t)=\psi_{h}(t)$ on $\left[-T_{0}, 0\right]$ has exactly one solution $z$ : $\left[-T_{0}, T\right] \rightarrow \mathbb{R}^{P}$ provided that the initial data satisfy the consistency condition $\boldsymbol{A}[\boldsymbol{C}]$.

Proof Problem (11) can be written in the following form

$$
\left\{\begin{aligned}
z_{D}^{\prime}-K_{1} z_{D}-K_{2} z_{A} & =\tilde{\varphi}_{D} \\
-K_{3} z_{D}-K_{4} z_{A} & =\tilde{\varphi}_{A}
\end{aligned}\right.
$$

with the initial conditions $z_{D}=\left(\psi_{h}\right)_{D}, \quad z_{A}=\left(\psi_{h}\right)_{A}$. From DA-irreducibility of the matrix $K$ it follows that $\operatorname{det}\left(K_{4}\right) \neq 0$. Hence

$$
z_{A}=K_{4}^{-1}\left(-K_{3} z_{D}-\tilde{\varphi}_{A}\right)
$$

Therefore

$$
z_{D}^{\prime}-\left(K_{1}-K_{2} K_{4}^{-1} K_{3}\right) z_{D}=-K_{2} K_{4}^{-1} \tilde{\varphi}_{A}+\tilde{\varphi}_{D} .
$$

In this theorem we only consider the case when the right hand side is dependent only on $t$. Hence from the boundedness, DA-irreducibility and continuity of $K$ there exist a unique solution of problem (12) (see [28] Thm VII). The component $z_{A}$ is uniquely determined by $z_{D}$. The consistency condition $\mathbf{A}[\mathbf{C}]$ guarantees the continuity of the solution. 
Lemma 2 Suppose that $\mathbf{A}[\sigma], \mathbf{A}[\mathbf{K}], \mathbf{A}[\mathbf{C}]$ are satisfied and $\left|g_{i}(t, \xi)\right| \leq \sigma(t,\|\xi\|)$ for $i \leq p$ and $g_{i}(t, \xi)=0$ for $i>p$. Then

$$
\|z(t)\| \leq \omega(t ; \gamma, \tilde{\gamma}) \text { on }\left[-T_{0}, T\right] \times \tilde{\Omega}_{h},
$$

where $z$ is a solution of

$$
\left[\begin{array}{cc}
I_{p \times p} & 0 \\
0 & 0
\end{array}\right] \frac{d}{d t} z-\boldsymbol{K} z=g\left(t, z_{t}\right)+\tilde{\Gamma}(t)
$$

with the initial condition (9) and $\gamma=\left\|\psi_{h}\right\| \tilde{\gamma}=\|\tilde{\Gamma}\|$.

Proof If $\tilde{\gamma}, \gamma \equiv 0$, then according to [11] $z \equiv 0$. If we assume that $\tilde{\gamma}, \gamma \geq 0$ then we consider the comparison system

$$
\begin{aligned}
x_{i}^{\prime} & =B_{i} \sum_{j=1, j \neq i}^{p} k_{i j} \frac{x_{j}}{B_{j}}+B_{i}\left\{\sigma\left(t, \sup _{1 \leq l \leq p}\left|\frac{x_{l}}{B_{l}}\right|_{t}+C_{K} \tilde{\gamma}\right)+\tilde{\gamma}\right\}, \quad i \leq p \\
\left|\frac{x_{i}}{B_{i}}\right| & \leq \sup _{1 \leq l \leq p}\left|\frac{x_{l}}{B_{l}}\right|_{t}+C_{K} \tilde{\gamma}, \quad i>p,
\end{aligned}
$$

where $\left|z_{i}\right| \leq \frac{x_{i}}{B_{i}}, C_{K}=\sup _{p+1 \leq i \leq P}\left(\sum_{l=1}^{p} k_{i l}\right)^{-1}$ and $B_{i}$ is the solution of the ODE

$$
B_{i}^{\prime}=-k_{i i} B_{i}, \quad B_{i}(0)=1 .
$$

Proceeding analogously like in [11] we have $x_{i}=x_{1} \frac{B_{i}}{B_{1}}, i=2, \ldots, P$ and

$$
\left[\frac{x_{1}}{B_{1}}\right]^{\prime} \leq \sigma\left(t,\left\|\frac{x_{1}}{B_{1}} \mathbf{1}\right\|_{t}+C_{K} \tilde{\gamma}\right)+\tilde{\gamma}
$$

It follows from the comparison principle for ODE's that

$$
\frac{x_{i}}{B_{i}}=\frac{x_{1}}{B_{1}} \leq \omega(t ; \gamma, \tilde{\gamma})-C_{K} \tilde{\gamma} \leq \omega(t ; \gamma, \tilde{\gamma}),
$$

which completes the proof.

The following lemma is crucial in the proof of Theorem 1.

Lemma $\mathbf{3}$ Suppose that $\mathbf{A}[\sigma], \mathbf{A}[\mathbf{K}], \mathbf{A}[\mathbf{C}]$ are satisfied and

$$
\left|\varphi_{i . h}(t, w)-\varphi_{i . h}(t, \tilde{w})\right| \leq \sigma(t,\|w-\tilde{w}\|) \text { for } i=1, \ldots, p
$$

and $\varphi_{i . h}(t, w)=0$ for $i=p+1, \ldots, P$. Then there exists exactly one solution of problem $(8,9)$. 
Proof Consider an iterative method, which starts from a prescribed function $\mathbf{u}_{0} \in$ $C\left(\left[-T_{0}, T\right], \mathbb{R}^{P}\right)$ satisfying (8) with some error $\tilde{\Gamma}(t)$ and $\tilde{\gamma}=\|\tilde{\Gamma}\|$. Now we consider the linear system of equations for each $k=0,1, \ldots$

$$
\left[\begin{array}{cc}
I_{p \times p} & 0 \\
0 & 0
\end{array}\right] \frac{d}{d t} \mathbf{u}_{k+1}-\mathbf{K} \mathbf{u}_{k+1}=\varphi_{h}\left(t,\left(\mathbf{u}_{k}\right)_{t}\right)
$$

with the initial condition (9). It follows from Lemma 1 that there is exactly one solution of the above problem. Applying Lemma 2 to the differences $\mathbf{u}_{k+l}-\mathbf{u}_{k}$ for $k, l \in \mathbb{N}$ we conclude that $\left\{\mathbf{u}_{k}\right\}_{k \in \mathbb{N}}$ is the Cauchy sequence.

\section{Stability and convergence}

We are now in position to state our main stability and convergence result for the MOL corresponding to $(1,2,3)$. We need the following assumptions on the functions $f, \beta$, $a_{i j}$, and the steps $h$ of the mesh.

\section{Assumption A}

- $f: Q \times C\left(Q_{0}\right) \times \mathbb{R}^{n} \rightarrow \mathbb{R}$ is continuous in $t, w, q$, the same property have the derivatives $\partial_{q_{j}} f$ and they are bounded,

- $a_{i j}: Q \rightarrow \mathbb{R}$ are bounded and continuous in $t$ for $i, j \in\{1, \ldots, n\}$,

$-\partial_{q_{j}} f, a_{i j}$ and steps $h$ satisfy the relations (CFL)

$$
\begin{gathered}
-\frac{h_{j}}{2}\left|\partial_{q_{j}} f\right|+a_{j j}-\sum_{l \neq j} \frac{h_{j}}{h_{l}}\left|a_{j l}\right| \geq 0 \\
a_{i j}\left(t, x^{(m)}\right) \leq 0 \text { for }(i, j) \in J_{-}, \quad a_{i j}\left(t, x^{(m)}\right) \geq 0 \text { for }(i, j) \in J_{+},
\end{gathered}
$$

$-|f(t, x, \bar{w}, q)-f(t, x, w, q)| \leq \sigma(t,\|\bar{w}-w\|),|f(t, x, 0,0)| \leq M_{f}$,

$-\beta$ is a bounded, continuous function such that $\beta \geq 0$.

Theorem 1 Suppose that $\mathbf{A}[\sigma]$, A are satisfied. Then there exists exactly one solution $u:\left[T_{0}, T\right] \rightarrow \tilde{\Omega}_{h} \mathbb{R}$ of problem $(5-7)$.

Proof Consider the iterative method. We choose an arbitrary function $\mathbf{u}_{0} \in$ $C\left(\left[-T_{0}, T\right], \mathbb{R}^{P}\right)$. Consider the ODE system

$$
\frac{d}{d t} u_{k}^{(m)}(t)-\sum_{i, j=1}^{n} a_{i j}\left(t, x^{(m)}\right) \delta_{i j}^{(2)} u_{k}^{(m)}(t)=f\left(t, x^{(m)},\left(\mathcal{J}_{h}\left[u_{k-1}\right]\right)_{t}, \delta u_{k}^{(m)}(t)\right)
$$

with the initial and boundary conditions (6)-(7).

We apply the Hadamard mean value theorem to the difference

$$
f\left(t, x^{(m)},\left(\mathcal{J}_{h}\left[u_{k-1}\right]\right)_{t}, \delta u_{k}^{(m)}(t)\right)-f\left(t, x^{(m)},\left(\mathcal{J}_{h}\left[u_{k-1}\right]\right)_{t}, \delta u_{k-1}^{(m)}(t)\right) .
$$


We have

$$
\begin{aligned}
& \frac{d}{d t} u_{k}^{(m)}(t)-\sum_{i, j=1}^{n} a_{i j}\left(t, x^{(m)}\right) \delta_{i j}^{(2)} u_{k}^{(m)}(t)-\sum_{i=1}^{n} \int_{0}^{1} \partial_{q_{i}} f(\Phi(\tau)) d \tau \delta_{i} u_{k}^{(m)}(t) \\
& \quad=f\left(t, x^{(m)},\left(\mathcal{J}_{h}\left[u_{k-1}\right]\right)_{t}, \delta u_{k-1}^{(m)}(t)\right)-\sum_{i=1}^{n} \partial_{q_{i}} f(\Phi(\tau)) d \tau \delta_{i} u_{k-1}^{(m)}(t),
\end{aligned}
$$

where

$$
\Phi_{0}(\tau)=\left(t, x^{(m)},\left(J_{h}\left[u_{k-1}\right]\right)_{t},(1-\tau) \delta u_{k-1}^{(m)}(t)+\tau \delta u_{k}^{(m)}(t)\right)
$$

We substitute the formulas for $\delta, \delta^{(2)}$ in the above equation. The matrix $\mathbf{K}$ consists of elements which are linear combinations of $a_{i j}$ and $\partial_{q_{j}} f$.

According to Lemma 2 we show that the matrix $\mathbf{K}$ satisfies all conditions of $\mathbf{A}[\mathbf{K}]$. Put

$$
\begin{aligned}
S_{0} & =\sum_{(i, j) \in J} \frac{1}{h_{i} h_{j}}\left|a_{i j}\left(t, x^{(m)}\right)\right|-2 \sum_{i=1}^{n} \frac{1}{h_{i}^{2}} a_{i i}\left(t, x^{(m)}\right), \\
S_{i}^{+} & =\frac{1}{2 h_{i}} \int_{0}^{1} \partial_{q_{i}} f(\Phi(\tau)) d \tau+\frac{1}{h_{i}^{2}} a_{i i}\left(t, x^{(m)}\right)-\sum_{j=1, j \neq i}^{n} \frac{\mid a_{i j}\left(t, x^{(m)} \mid\right.}{h_{i} h_{j}}, \\
S_{i}^{-} & =-\frac{1}{2 h_{i}} \int_{0}^{1} \partial_{q_{i}} f(\Phi(\tau)) d \tau+\frac{1}{h_{i}^{2}} a_{i i}\left(t, x^{(m)}\right)-\sum_{j=1, j \neq i}^{n} \frac{\mid a_{i j}\left(t, x^{(m)} \mid\right.}{h_{i} h_{j}}, \\
S_{i j} & =\frac{1}{2 h_{i} h_{j}}\left|a_{i j}\left(t, x^{(m)}\right)\right| .
\end{aligned}
$$

It follows from assumption $\mathbf{A}$ that

$$
S_{0} \leq 0, \quad S_{i}^{+}, S_{i}^{-} \geq 0, \quad S_{i j} \geq 0, \quad i, j=1, \ldots, n
$$

and

$$
S_{0}+\sum_{i=1}^{n}\left(S_{i}^{+}+S_{i}^{-}\right)+2 \sum_{(i, j) \in J} S_{i j}=0 .
$$

Since $\mathcal{J}_{h}[u]$ is a convex combination of $u\left(t, x^{(m)}\right)$ and

$$
\exp \left\{-\left\|x^{(m)}-r\left(x^{(m)}\right)\right\| \beta\left(t, R\left(x^{(m)}\right)\right\} \leq 1,\right.
$$

first two conditions of $\mathbf{A}[\mathbf{K}]$ are satisfied. Note that at least one coefficient in $\mathcal{J}_{h}$ is positive. Thus last two conditions of $\mathbf{A}[\mathbf{K}]$ are satisfied. It follows from Lemma 1 that there is exactly one solution of problem (13). 
Next we analyse the difference $\mathbf{u}_{k+l}-\mathbf{u}_{k}$ for $k, l \in \mathbb{N}$. Put $\Delta_{l} u=\mathbf{u}_{k+l}-\mathbf{u}_{k}$. We have

$$
\begin{aligned}
& \frac{d}{d t} \Delta_{l} u_{k}^{(m)}-\sum_{i, j=1}^{n} a_{i j}\left(t, x^{(m)}\right) \delta_{i j}^{(2)} \Delta_{l} u_{k}^{(m)}(t) \\
& \quad=f\left(t, x^{(m)},\left(\mathcal{J}_{h}\left[u_{k+l-1}\right]\right)_{t}, \delta u_{k+l}^{(m)}(t)\right)-f\left(t, x^{(m)},\left(\mathcal{J}_{h}\left[u_{k-1}\right]\right)_{t}, \delta u_{k}^{(m)}(t)\right) .
\end{aligned}
$$

We apply the Hadamard mean value theorem

$$
\begin{aligned}
& \frac{d}{d t} \Delta_{l} u_{k}^{(m)}-\sum_{i, j=1}^{n} a_{i j}\left(t, x^{(m)}\right) \delta_{i j}^{(2)} \Delta_{l} u_{k}^{(m)}(t)-\sum_{i=1}^{n} \int_{0}^{1} \partial_{q_{i}} f(\Upsilon(\tau)) d \tau \delta_{i} \Delta_{l} u_{k}^{(m)} \\
& =f\left(t, x^{(m)},\left(\mathcal{J}_{h}\left[u_{k+l-1}\right]\right)_{t}, \delta u_{k}^{(m)}(t)\right)-f\left(t, x^{(m)},\left(\mathcal{J}_{h}\left[u_{k-1}\right]\right)_{t}, \delta u_{k}^{(m)}(t)\right),
\end{aligned}
$$

where

$$
\Upsilon(\tau)=\left(t, x^{(m)},\left(J_{h}\left[u_{k+l-1}\right]\right)_{t},(1-\tau) \delta u_{k}^{(m)}(t)+\tau \delta u_{k+l}^{(m)}(t)\right) .
$$

Applying Lemma 2 we have

$$
\left\|\Delta_{l} u_{k}(t)\right\| \leq \omega_{k}(t, \gamma, \tilde{\gamma})
$$

where

$$
\lim _{k \rightarrow \infty} \omega_{k}(t)=0
$$

uniformly on $[0, \tilde{T}]$, for each $\tilde{T} \in(0, T)$ (see [21]). We conclude that $\left\{\mathbf{u}_{k}\right\}_{k \in \mathbb{N}}$ is the Cauchy sequence.

Theorem 2 Suppose that $\mathbf{A}[\sigma], \boldsymbol{A}$ are satisfied and

$-u$ is a solution of $(1,2,3)$ and $\tilde{u}$ is a solution of $(5,6,7)$ such that

$$
\begin{aligned}
& \left|u^{(m)}(t)-\tilde{u}^{(m)}(t)\right| \leq \gamma_{h} \text { on }\left[-T_{0}, 0\right] \times \tilde{\Omega}_{h}, \\
& \left|u^{(m)}(t)-\tilde{u}^{(m)}(t)\right| \leq \gamma_{h}^{*}(t) \text { on }(t, x) \in[0, T] \times \Omega_{h}^{*} .
\end{aligned}
$$

Then there is $\omega_{h}:\left[-T_{0}, T\right] \rightarrow \mathbb{R}_{+}$such that

$$
\left|u^{(m)}(t)-\tilde{u}^{(m)}(t)\right| \leq \omega_{h}(t), \text { and } \lim _{\|h\| \rightarrow 0} \omega_{h}(t)=0 .
$$

Remark 1 We assume that sign $a_{i j}$ is constant. One can omit this assumption by considering $J_{-}, J_{+}$as sets which depend on $x^{(m)}$. 
Proof (Theorem 2) Let $\Gamma_{h}: Q_{h} \rightarrow \mathbb{R}$ be defined by the relation

$$
\begin{aligned}
& \frac{d}{d t} u^{(m)}(t)-\sum_{i, j=1}^{n} a_{i j}\left(t, x^{(m)}\right) \delta_{i j}^{(2)} u^{(m)}(t) \\
& \quad=f\left(t, x^{(m)},\left(\mathcal{J}_{h}[u]\right)_{t}, \delta u^{(m)}(t)\right)+\Gamma_{h}^{(m)}(t) \text { on } Q_{h}
\end{aligned}
$$

It follows that there is $\tilde{\gamma}_{h}$ such that

$$
\left|\Gamma_{h}^{(m)}(t)\right| \leq \tilde{\gamma}_{h} \quad \text { on } Q_{h} \quad \text { and } \lim _{h \rightarrow 0} \tilde{\gamma}_{h}=0
$$

From the definition of $\mathcal{J}_{h}$ we have

$$
\left|\mathcal{J}_{h}[u]-\mathcal{J}_{h}[\tilde{u}]\right| \leq\|u-\tilde{u}\| \leq \gamma_{h} \quad \text { on } \quad\left[-T_{0}, 0\right] \times \Omega_{h}^{*} .
$$

Applying the Hadamard mean value theorem we have

$$
\begin{aligned}
& \frac{d}{d t}(u-\tilde{u})^{(m)}(t)-\sum_{i, j=1}^{n} a_{i j}\left(t, x^{(m)}\right) \delta_{i j}^{(2)}(u-\tilde{u})^{(m)}(t) \\
& \quad-\sum_{i=1}^{n} \int_{0}^{1} \partial_{q_{i}} f(\Phi(\tau)) d \tau\left[\delta_{i}(u-\tilde{u})^{(m)}(t)\right] \\
& =f\left(t, x^{(m)},\left(\mathcal{J}_{h}[u]\right)_{t}, \delta \tilde{u}^{(m)}(t)\right) \\
& \quad-f\left(t, x^{(m)},\left(\mathcal{J}_{h}[\tilde{u}]\right)_{t}, \delta \tilde{u}^{(m)}(t)\right)+\Gamma_{h}^{(m)}
\end{aligned}
$$

where

$$
\Phi(\tau)=\left(t, x^{(m)},\left(\mathcal{J}_{h}[u]\right)_{t}, \delta \tilde{u}^{(m)}(t)+\tau\left(\delta u^{(m)}(t)-\delta \tilde{u}^{(m)}(t)\right)\right) .
$$

Hence the above equation can be written in the following form

$$
\left[\begin{array}{cc}
I_{p \times p} & 0 \\
0 & 0
\end{array}\right] \frac{d}{d t}(u-\tilde{u})-\mathbf{K}(u-\tilde{u})=g\left(t,\left(\mathcal{J}_{h}[(u-\tilde{u})]\right)_{t}\right)+\Gamma_{h}(t)
$$

where

$$
\Gamma_{i . h}(t)= \begin{cases}\Gamma_{i . h}(t), & i=1, \ldots, p \\ \gamma_{h}^{*}, & i=p+1, \ldots, P .\end{cases}
$$

According to Lemma 2 we show that the matrix $\mathbf{K}$ satisfies all conditions of $\mathbf{A}[\mathbf{K}]$.

Put 


$$
\begin{aligned}
S_{0} & =\sum_{(i, j) \in J} \frac{1}{h_{i} h_{j}}\left|a_{i j}\left(t, x^{(m)}\right)\right|-2 \sum_{i=1}^{n} \frac{1}{h_{i}^{2}} a_{i i}\left(t, x^{(m)}\right), \\
S_{i}^{+} & =\frac{1}{2 h_{i}} \int_{0}^{1} \partial_{q_{i}} f(\Phi(\tau)) d \tau+\frac{1}{h_{i}^{2}} a_{i i}\left(t, x^{(m)}\right)-\sum_{j=1, j \neq i}^{n} \frac{\mid a_{i j}\left(t, x^{(m)} \mid\right.}{h_{i} h_{j}}, \\
S_{i}^{-} & =-\frac{1}{2 h_{i}} \int_{0}^{1} \partial_{q_{i}} f(\Phi(\tau)) d \tau+\frac{1}{h_{i}^{2}} a_{i i}\left(t, x^{(m)}\right)-\sum_{j=1, j \neq i}^{n} \frac{\mid a_{i j}\left(t, x^{(m)} \mid\right.}{h_{i} h_{j}}, \\
S_{i j} & =\frac{1}{2 h_{i} h_{j}}\left|a_{i j}\left(t, x^{(m)}\right)\right| .
\end{aligned}
$$

It follows from assumption $\mathbf{A}$ that

$$
S_{0} \leq 0, \quad S_{i}^{+}, S_{i}^{-} \geq 0, \quad S_{i j} \geq 0, \quad i, j=1, \ldots, n
$$

and

$$
S_{0}+\sum_{i=1}^{n}\left(S_{i}^{+}+S_{i}^{-}\right)+2 \sum_{(i, j) \in J} S_{i j}=0 .
$$

Since $\mathcal{J}_{h}[u]$ is a convex combination of $u\left(t, x^{(m)}\right)$ and

$$
\exp \left\{-\left\|x^{(m)}-r\left(x^{(m)}\right)\right\|\right) \beta\left(t, R\left(x^{(m)}\right)\right\} \leq 1
$$

first two conditions of $\mathbf{A}[\mathbf{K}]$ are satisfied. Note that at least one coefficient in $\mathcal{J}_{h}$ is positive. Thus last two conditions of $\mathbf{A}[\mathbf{K}]$ are satisfied. The conclusion in (14) can be seen by observing that the function $\omega:\left[-T_{0}, T\right] \times H \rightarrow \mathbb{R}_{+}$is a solution of the Cauchy problem

$$
\omega^{\prime}(t)=\sigma(t, \omega(t))+\tilde{\gamma}_{h}, \quad \omega(t)=\gamma_{h}, \text { for } t \in\left[-T_{0}, 0\right] .
$$

\section{Numerical examples}

We apply the results presented in Sect. 3 to a differential equation with deviated variables and to a differential integral problem. We consider our numerical examples on the cylinder $[0, T] \times B_{1}$, where $B_{1}$ is the unit ball centered at $(0,0)$.

Example 1 Consider the differential integral problem

$$
\begin{aligned}
& \partial_{t} u-\partial_{x x}^{2} u+\frac{1}{2} \partial_{x y}^{2} u-\partial_{y y}^{2} u=\left(1+x^{2}+y^{2}\right) \int_{0}^{t} u(s, x, y) d s \\
& \quad+\left(4 x^{2} t^{2}-2 x y t^{2}+4 y^{2} t^{2}\right) u+\left(4 t-2-x^{2}-y^{2}\right) \sin \left(t\left(x^{2}+y^{2}+1\right)\right),
\end{aligned}
$$


Table 1 Maximal error for PDE with integrals

\begin{tabular}{llllll}
\hline$t^{(r)}$ & 0.050000 & 0.100000 & 0.150000 & 0.200000 & 0.250000 \\
$\varepsilon_{\text {max }}^{(r)}$ & 0.0004152 & 0.0008028 & 0.0033388 & 0.0074885 & 0.0132798 \\
\hline
\end{tabular}

Table 2 Maximal error for PDE with delays

\begin{tabular}{llllll}
\hline$t^{(r)}$ & 0.100000 & 0.200000 & 0.300000 & 0.400000 & 0.500000 \\
$\varepsilon_{\max }^{(r)}$ & 0.0078578 & 0.0137890 & 0.0174465 & 0.0182035 & 0.0184644 \\
\hline
\end{tabular}

with initial and boundary conditions

$$
\begin{aligned}
u(0, x, y) & =1 \text { on } B_{1}, \\
\frac{\partial u}{\partial n}+2 t \tan \left(t\left(x^{2}+y^{2}+1\right)\right) u & =0 \quad \text { on }\left[0, \frac{\pi}{8}\right] \times \partial B_{1} .
\end{aligned}
$$

The solution of the above problem is known, $u(t, x, y)=\cos \left(t\left(x^{2}+y^{2}+1\right)\right)$.

In Table 1 we give experimental values of the maximal error $\varepsilon_{\max }$ for $h_{0}=0.01$ and $h_{1}=h_{2}=0.125$, where $\left(h_{0}, h_{1}, h_{2}\right)$ are steps of the mesh with respect to $(t, x, y)$.

Example 2 Consider the differential equation with deviated variables

$$
\begin{aligned}
\partial_{t} u_{t} & -\partial_{x x}^{2} u+\frac{1}{2} \partial_{x y}^{2} u-\partial_{y y}^{2} u=u\left(\frac{t}{2}, x, y\right) \\
& +\left[-x^{2}-y^{2}+4 t-4 t^{2} x^{2}-2 t^{2} x y-4 t^{2} y^{2}\right] u-\exp \left\{-0.5 t\left(x^{2}+y^{2}\right)\right\}
\end{aligned}
$$

with initial and boundary conditions

$$
\begin{aligned}
& u(0, x, y)=1 \text { on } B_{1}, \\
& \frac{\partial u}{\partial n}+2 t u=0 \text { on }[0,1] \times \partial B_{1} .
\end{aligned}
$$

The solution of the above problem is known, $u=e^{-t\left(x^{2}+y^{2}\right)}$.

In Table 2 we give experimental values of the maximal error for $h_{0}=0.01$ and $h_{1}=h_{2}=0.125$, where $\left(h_{0}, h_{1}, h_{2}\right)$ are steps of the mesh with respect to $(t, x, y)$.

The above examples are carried out for two-dimensional spatial variables. This is done only for our convenience of implementation. The theory presented in our paper is not limited with respect to the dimension of spatial variables. Both coefficients of the derivatives of the unknown function and the functions appearing on the right hand side of the equation and the initial and the boundary conditions satisfy the assumptions imposed in our paper. The computed results have been compared with the exact solution to show the required accuracy of the method. The computation time is $0.38 \mathrm{sec}$ for the 
first example, and $24.82 \mathrm{~s}$ for the second example. The presented experiments illustrate the convergence of the proposed method.

Acknowledgments The authors would like to thank the anonymous referees for their valuable coments and suggestions.

Open Access This article is distributed under the terms of the Creative Commons Attribution License which permits any use, distribution, and reproduction in any medium, provided the original author(s) and the source are credited.

\section{References}

1. Abdulla, U.G.: On the dirichlet problem for reaction-diffusion equations in non-smooth domains. Nonlinear Anal. 47, 765-776 (2001)

2. Al'shin, A.B., Al'shina, E.A., Kalitkin, N.N., Koryagina, A.B.: Numerical solution of superstiff differential-algebraic systems. Dokl. Math. 73(3), 460-464 (2006)

3. Ascher, U.M., Petzold, L.R.: Computer methods for ordinary differential equations and differentialalgebraic equations. SIAM (1998)

4. Dehghan, M., Shakeri, F.: Method of lines solutions of the parabolic inverse problem with an overspecification at a point. Numer. Algorithms 50,417-437 (2009)

5. Fakhar-Izadi, F., Dehghan, M.: Space-time spectral method for a weakly singular parabolic partial integro-differential equation on irregular domains. Comput. Math. Appl. 67, 1884-1904 (2014)

6. Hairer, E., Wanner, G.: Solving ordinary differential equation. In: Stiff and differential-algebraic problems. Springer, Berlin (Mir Moscow 2 (1999)) (1996)

7. Hale, J.K., Verduyn Lunel, S.M.: Introduction to Functional Differential Equations. Springer, New York (1993)

8. Hundsdorfer, W., Verwer, J.G.: Numerical solution of time-dependent advection-diffusion-reaction equations. Springer, Berlin (2003)

9. Incropera, F.P., DeWitt, D.P.: Fundamentals of heat and mass transfer. John Wiley (2007)

10. Lekomtsev, A.V., Pimenov, V.G.: A semiexplicit method for numerical solution of functional differential algebraic equations. Russ Math 53(5), 54-58 (2009)

11. Leszczyński, H.: Comparison ODE theorems related to the method of lines. J. Appl. Anal. 17(1), 137-154 (2011)

12. Kamont, Z.: Hyperbolic functional differential inequalities and applications. Kluwer Academic Publishers, Dordrecht (1999)

13. Koleva, M.N., Vulkov, L.G.: Quasilinearization numerical scheme for fully nonlinear parabolic problems with applications in models of mathematical finance. Math. Comput. Model. 57(9-10), 2564-2575 (2013)

14. Kropielnicka, K.: Implicit difference methods for parabolic FDE on cylindrical domains. Dynam. Syst. Appl. 19(3-4), 557-576 (2010)

15. Kunkel, P., Mehrmann, V.: Differential-algebraic equations: analysis and numerical solution, European Mathematical Society (2006)

16. Mittal, R.C., Jiwari, R.: A higher order numerical scheme for some nonlinear differential equations. Models in biology. Int. J. Comput. Meth. Eng. Sci. Mech. 12(3), 134-140 (2011)

17. Mitchell, A.R., Griffiths, D.F.: The finite difference method in partial differential equations. Wiley, New York (1980)

18. Mohebbia, A., Dehghan, M.: High-order scheme for determination of a control parameter in an inverse problem from the over-specified data. Comput. Phy. Commun. 181, 1947-1954 (2010)

19. Morton, K.W., Mayers, D.F.: Numerical solution of partial differential equations. Cambridge University Press, Cambridge (1994)

20. Murray, J.D.: Mathematical biology. Springer, Berlin (1993)

21. Netka, M.: Differential difference inequalities related to parabolic functional differential equations. Opuscula Math. 30(1), 95-115 (2010) 
22. Papac, J., Helgadottir, A., Ratsch, C., Gibou, F.: A level set approach for diffusion and Stefan-type problems with Robin boundary conditions on quadtree/octree adaptive Cartesian grids. J. Comput. Phys. 233, 241-261 (2013)

23. Pao, C.V.: Nonlinear parabolic and elliptic equations. Plenum Press, New York (1992)

24. Shakeri, F., Dehghan, M.: The method of lines for solution of the one-dimensional wave equation subject to an integral conservation condition. Comput. Math. Appl. 56, 2175-2188 (2008)

25. Schiesser, W.E.: The numerical method of lines. Integration of partial differential equations. Academic Press Inc., San Diego (1991)

26. Soltaniana, F., Dehghanb, M., Karbassia, S.M.: Solution of the differential algebraic equations via homotopy perturbation method and their engineering applications. Int. J. Comput. Math. 87, 19501974 (2010)

27. Vande Wouwer, A., Saucez, Ph, Schiesser, W.E.: Adaptive method of lines. Capman \& Hall/CRC, USA (2001)

28. Walter, W.: Ordinary differential equations. Springer, New York (1998)

29. Wrzosek, D.: Global attractor for a chemotaxis model with prevention of overcrowding. Nonlinear Anal. Theory Methods Appl. 59(8), 1293-1310 (2004)

30. Zubik-Kowal, B.: The method of lines for parabolic differential-functional equations. IMA J. Numer. Anal. 17(1), 103-123 (1997) 